\title{
ZARISKI-DENSITY OF EXCEPTIONAL SETS FOR HYPERGEOMETRIC FUNCTIONS
}

\author{
PIERRE-ANTOINE DESROUSSEAUX, MARVIN D. TRETKOFF, \\ AND PAULA TRETKOFF
}

\begin{abstract}
We extend the results of Desrousseaux in [10], [11], [12]. In those papers an exceptional set was constructed for the AppellLauricella hypergeometric functions associated to rational ball tuples. The definition of this set requires quite subtle conditions necessary for the application of the André-Oort conjecture. In this paper, we show that generalizations of the André-Oort conjecture by Pink [20] lead to similar results for a more natural exceptional set, namely the set of algebraic points at which the function takes algebraic values. Desrousseaux's exceptional set is in general a proper subset of this set.
\end{abstract}

\section{INTRODUCTION}

Wüstholz's analytic subgroup theorem [31], [32], [33] implies that linear dependence relations over $\overline{\mathbb{Q}}$ between periods on algebraic curves defined over $\overline{\mathbb{Q}}$ can only arise from isogenies between the Jacobians of the curves. This theorem was applied in [29] to obtain the first significant results about the transcendence of the value of the classical hypergeometric function of one variable when that variable assumes an algebraic number. In [8], questions of this type were related to the André-Oort conjecture: a closed subvariety $Z$ of a Shimura variety $S$ that contains a Zariski-dense subset $\mathcal{T}$ of the special points of $S$ is the union of special subvarieties. (Recall that a special subvariety of a Shimura variety $S$ is defined to be an irreducible component of a Shimura subvariety or of its image under a Hecke operator. See [18] for a survey of recent progress concerning the André-Oort conjecture.) In fact, the results obtained in [8] only require a particular case of the André-Oort conjecture: $Z$ is a curve and the special points of $\mathcal{T}$ all lie in a single Hecke orbit. This particular case was proved in [14].

In [10], [11], [12], the results of [8] and [29] were extended to several complex variables, with the Appell-Lauricella hypergeometric functions playing

Typeset February 12, 2006 [8:05].

1991 Mathematics Subject Classification. 11J91, 33C65.

Key words and phrases. Hypergeometric functions, transcendence, Shimura varieties.

Paula Tretkoff (formerly known by her maiden name "Paula Cohen") acknowledges support from NSF grant number DMS-0400942. 
the role of the classical (Gauss) hypergeometric function of one variable. This passage to several variables requires that we utilize a proper subset of the set of algebraic points at which the function takes algebraic values. The definition of this smaller set requires quite subtle conditions. The results so obtained depend on the validity of a particular case of the André-Oort conjecture when the dimension of $Z$ is greater than one. Neither the general André-Oort conjecture nor the relevant particular case has yet been proved.

At the heart of this paper is our observation that, in light of the analytic subgroup theorem, the set of algebraic points at which an Appell-Lauricella function takes on algebraic values leads to consideration of Hecke orbits of special subvarieties that may be of positive dimension. This, in turn, raises the question of how to extend the André-Oort conjecture if the special points in $\mathcal{T}$ are replaced by special subvarieties, or more particularly by a single Hecke orbit of a special subvariety. This type of problem is studied by Pink in $[20]$.

On the other hand, in general it is necessary to impose extra conditions of the type considered by Desrousseaux on the exceptional set in order to apply the original André-Oort conjecture. In that case we only consider Zariski-density of sets $\mathcal{T}$ of special points, rather than of sets of varieties of higher dimension.

Throughout this paper, we limit ourselves to hypergeometric functions associated with a rational ball tuple (condition (2.1)). This ensures that the differential form in (2.3) is of the first kind. It should be possible to treat rational tuples not satisfying (2.1) similarly. For example, the corollaries of the analytic subgroup theorem that we utilize also apply to differentials of the second kind.

This paper is organized as follows. In $\S 2$ we state some conjectures of Pink and our main applications of these conjectures to exceptional values of hypergeometric functions. In $\S 3$ we construct Shimura varieties related to these functions. In $\S 4$ we interpret the exceptional set as isogeny classes of an analytic family of abelian varieties. This is accomplished using transcendence methods. Our main results are established in $\S \S 5$ and 6 . Finally, we conclude with some examples in $\S 7$.

\section{Statement of Results}

The Appell-Lauricella hypergeometric functions of several variable are generalizations of the classical hypergeometric function of one variable, see [AK], Chapter VII. Appell's name is usually associated to the two variable case, whereas the case of more variables is usually ascribed to Lauricella. These functions are defined on the weighted configuration space of $n$ distinct points on the projective line. The weights are in general given by $(n+3)$ real numbers. We assume throughout this note that these weights $\mu=\left\{\mu_{i}\right\}_{i=0}^{n+2}$ 
are rational numbers satisfying the ball $(n+3)$-tuple condition of [9]:

$$
0<\mu_{i}<1, \quad i=0, \ldots, n+2, \quad \sum_{i=0}^{n+2} \mu_{i}=2 .
$$

We refer to such weights as rational ball tuples.

Matching the indices of the variables $x_{i}$ with those of the weights $\mu_{i}$, we write the space of regular points for these functions as

$$
\mathcal{Q}=\left\{\mathbf{x}=\left(x_{2}, \ldots, x_{n+1}\right) \in \mathbb{P}_{1}(\mathbb{C})^{n}: x_{i} \neq 0,1, \infty ; \quad x_{i} \neq x_{j}, i \neq j\right\} .
$$

This space has the structure of a quasi-projective variety over $\overline{\mathbb{Q}}$ and can be identified with

$$
\mathcal{Q}^{\prime}=\left\{\left(x_{0}, x_{1}, x_{2}, \ldots, x_{n+1}, x_{n+2}\right) \in \mathbb{P}_{1}(\mathbb{C})^{n+3}: x_{k} \neq x_{l} ; k \neq l\right\} / \operatorname{Aut}\left(\mathbb{P}_{1}\right)
$$

where $\operatorname{Aut}\left(\mathbb{P}_{1}\right)$ acts diagonally and freely.

The Appell-Lauricella hypergeometric functions are solutions of a system $\mathcal{H}_{\mu}$ of linear partial differential equations in $n$ variables $x_{i}, i=2, \ldots n+1$ with regular singularities along $x_{i}=0,1, \infty, x_{i}=x_{j}(j \neq i$ ) (see for example [28]). The system $\mathcal{H}_{\mu}$ has an $(n+1)$-dimensional solution space. We denote by $\Delta_{\mu}$ its monodromy group. It is a subgroup of $\mathrm{PU}(1, n)$. The $\mu$ for which $\Delta_{\mu}$ is a lattice in $\mathrm{PU}(1, n)$ have been computed (see [9]). When $n=1$, there are infinitely many such lattices, although only finitely many of them are arithmetic [27]. When $n=2$, there are 58 such lattices, of which 15 are not arithmetic. For $n$ between 3 and 12, there are 32 such lattices, with only 1 not being arithmetic. For $n \geq 13$, none of the monodromy groups is a lattice (see [9], [17], [21]).

There is a unique solution $F=F_{\mu}(\mathbf{x})$ of $\mathcal{H}_{\mu}$ which is holomorphic at the singular point $\mathbf{x}=\mathbf{0}$ and satisfies $F_{\mu}(\mathbf{0})=1$. This generalizes the classical hypergeometric function in one complex variable. For $\mathbf{x} \in \mathcal{Q}$, let $\omega_{1}$ be the differential form

$$
\omega_{1}=\omega_{1}(\mu ; \mathbf{x})=u^{-\mu_{0}}(u-1)^{-\mu_{1}} \prod_{i=2}^{n+1}\left(u-x_{i}\right)^{-\mu_{i}} d u .
$$

Although $\mathbf{x}=\mathbf{0} \notin \mathcal{Q}$, we also let,

$$
\omega_{1}(\mu ; \mathbf{0})=u^{-\mu_{0}-\sum_{i=2}^{n+1} \mu_{i}}(u-1)^{-\mu_{1}} d u .
$$

For $\mathbf{x}=\left(x_{2}, \ldots, x_{n+1}\right) \in \mathcal{Q}$, we have

$$
F=F_{\mu}(\mathbf{x})=\int_{1}^{\infty} \omega_{1}(\mu ; \mathbf{x}) / \int_{1}^{\infty} \omega_{1}(\mu ; \mathbf{0}),
$$

the normalizing constant ensuring that $F_{\mu}(\mathbf{0})=1$. With $B(\alpha, \beta)$ the classical Beta function, we may write this as,

$$
F=F_{\mu}(\mathbf{x})=B\left(1-\mu_{1}, 1-\mu_{n+2}\right)^{-1} \int_{1}^{\infty} \omega_{1}(\mu ; \mathbf{x}) .
$$


We define the exceptional set $\mathcal{E}_{F}$ of $F$ to be the subset of $\mathcal{Q}$ at which $F$ takes special values, that is,

$$
\mathcal{E}_{F}=\left\{\mathbf{x} \in \mathcal{Q} \cap \overline{\mathbb{Q}}^{n}: F_{\mu}(\mathbf{x}) \in \overline{\mathbb{Q}}^{*}\right\} .
$$

Several of our results will be conditional on the following consequences of more general of conjectures of Pink. Recall that a special subvariety of a Shimura variety $S$ is by definition an irreducible component of a Shimura subvariety, or of its image under a Hecke operator. The special closure of an irreducible subvariety $Z$ of $S$ is the smallest special subvariety containing $Z$. The special subvarieties of dimension zero are called the special points. For the Shimura varieties of this paper, they coincide with the CM (complex multiplication) points.

Conjecture 2.1. (see [20]) Consider a Shimura variety $S$ over $\mathbb{C}$ and an irreducible closed subvariety $Z$. Let $S_{Z}$ be the special closure of $Z$. Then the intersection of $Z$ with the union of all special subvarieties of $S$ of dimension strictly less than $\operatorname{dim}\left(S_{Z}\right)-\operatorname{dim}(Z)$ is not Zariski dense in $Z$.

In fact we only need this conjecture in the following weaker form:

Conjecture 2.2. Consider a Shimura variety $S$ over $\mathbb{C}$ and an irreducible closed subvariety $Z$. Let $S_{Z}$ be the special closure of $Z$. Then the intersection of $Z$ with the Hecke orbit of a special subvariety of $S$ of dimension strictly less than $\operatorname{dim}\left(S_{Z}\right)-\operatorname{dim}(Z)$ is not Zariski dense in $Z$.

We can now state our main result:

Theorem 2.3. If $\mu_{1}+\mu_{n+2} \leq 1$, then $\mathcal{E}_{F}$ is empty. If $\mu_{1}+\mu_{n+2}>1$, and $\Delta_{\mu}$ is an arithmetic lattice in $\mathrm{PU}(1, n)$, then $\mathcal{E}_{F}$ is Zariski-dense in $\mathcal{Q}$. Moreover, if we assume the validity of Conjecture 2.2, then $\mathcal{E}_{F}$ is Zariskidense in $\mathcal{Q}$ only if $\Delta_{\mu}$ is an arithmetic lattice in $\mathrm{PU}(1, n)$.

In the case $n=1$, the particular case of the André-Oort conjecture proven in [14] gives Theorem 2.3 unconditionally, see [8], [29]. Wolfart [29] was the first to realize that the arithmetic nature of the monodromy group of the classical hypergeometric function of one variable intervened in the study of its exceptional set.

In the case $n=2$, and when the monodromy group is discrete, it turns out that the André-Oort conjecture is sufficient.

Theorem 2.4. Suppose that $n=2$, that $\mu_{1}+\mu_{4}>1$, and that $\Delta_{\mu}$ is a lattice in $\mathrm{PU}(1,2)$. If $\Delta_{\mu}$ is arithmetic, then $\mathcal{E}_{F}$ is Zariski-dense in $\mathcal{Q}$. Moreover, assume the validity of the André-Oort conjecture when the points of $\mathcal{T}$ are special points in a single Hecke orbit. Then $\mathcal{E}_{F}$ is Zariski-dense in $\mathcal{Q}$ only if $\Delta_{\mu}$ is arithmetic.

Our results should shed light on the problems raised in the work of the Cohen, Shiga, and Wolfart discussed in $§ 5$, [23] (see also [5]). 


\section{Preliminaries about Prym varieties}

In this section, we construct analytic families of abelian varieties associated with hypergeometric functions. Following [22], [24] (which relate to the case $n=1$ ), we call these abelian varieties Prym varieties rather than hypergeometric varieties as in [2] (see also [6], [7], [23]).

For two non-zero complex numbers $a, b$, we write $a \sim b$ if $a / b$ is algebraic. We say then that $a$ and $b$ are proportional over $\overline{\mathbb{Q}}$. Let $\mu=\left\{\mu_{i}\right\}_{i=0}^{n+2}$ be a rational ball $(n+3)$-tuple and let $N$ be the least common denominator of the $\mu_{i}$. Let $K=\mathbb{Q}(\zeta)$, where $\zeta=\exp (2 \pi i / N)$. For $s \in(\mathbb{Z} / N \mathbb{Z})^{*}$, let $\sigma_{s}$ be the Galois embedding of $K$ which maps $\zeta \mapsto \zeta^{s}$.

If $\mathbf{x} \in \mathcal{E}_{F}$, then by $(2.5)$ we have the relation between transcendental numbers

$$
B\left(1-\mu_{1}, 1-\mu_{n+2}\right) \sim \int_{1}^{\infty} \omega_{1}(\mu ; \mathbf{x}) .
$$

This implies that periods on certain (different) algebraic curves defined over $\overline{\mathbb{Q}}$ are proportional over $\overline{\mathbb{Q}}$.

Indeed, by (2.1), for $\mathbf{x} \in \mathcal{Q}$ the differential $\omega_{1}(\mu ; \mathbf{x})$ is of the first kind on a projective non-singular curve $\mathcal{C}_{N}=\mathcal{C}_{N}(\mathbf{x})$ with affine model

$$
w^{N}=u^{N \mu_{0}}(u-1)^{N \mu_{1}} \prod_{i=2}^{n+1}\left(u-x_{i}\right)^{N \mu_{i}} .
$$

Notice that for $\mathbf{x} \in \overline{\mathbb{Q}}^{n}$ this curve is defined over a number field. On the other hand $\omega_{1}(\mu ; \mathbf{0})$ is a differential form on a projective nonsingular curve $\mathcal{C}_{N}(\mathbf{0})$ with affine model

$$
w^{N}=u^{N \mu_{n+2}}(1-u)^{N \mu_{1}}
$$

which is the image of a morphism from a Fermat curve.

If $\mu_{1}+\mu_{n+2}<1$, then $B\left(1-\mu_{1}, 1-\mu_{n+2}\right)$ is proportional over $\overline{\mathbb{Q}}$ to a non-zero period of $\omega_{1}(\mu ; \mathbf{0})$, which is of the second kind. If $\mu_{1}+\mu_{n+2}=1$, then $B\left(1-\mu_{1}, 1-\mu_{n+2}\right)$ is proportional over $\overline{\mathbb{Q}}$ to $\pi$. (For more details see [16], [30]).

From now on, we assume that $\mu_{1}+\mu_{n+2}>1$. Then the non-zero transcendental number $B\left(1-\mu_{1}, 1-\mu_{n+2}\right)$ is proportional over $\overline{\mathbb{Q}}$ to a period of the first kind on $\mathcal{C}_{N}(\mathbf{0})$. For rational numbers $0<\alpha, \beta<1$, we let $T_{\alpha, \beta}$ be the system of representatives of $(\mathbb{Z} / N \mathbb{Z})^{*} /\{ \pm 1\}$ given by

$$
T_{\alpha, \beta}=\left\{s \in(\mathbb{Z} / N \mathbb{Z})^{*}:\langle s \alpha\rangle+\langle s \beta\rangle<1\right\},
$$

where for a real number $a$, let $[a]$ be the integer part of $a$ and let $\langle a\rangle=$ $a-[a]$ be the fractional part of $a$. The Prym variety of $\mathcal{C}_{N}(\mathbf{0})$ is defined as the connected component of the origin in the intersection over the proper divisors $f$ of $N$ of the kernel of the natural maps from $\operatorname{Jac}\left(\mathcal{C}_{N}(\mathbf{0})\right)$ to $\operatorname{Jac}\left(\mathcal{C}_{f}(\mathbf{0})\right)$. It is a principally polarized abelian variety $A_{1, n+2}^{\prime}$ of dimension $\varphi(N) / 2$, with endomorphism algebra containing $K$. It is a power of a simple 
abelian variety with CM (complex multiplication) in the sense of ShimuraTaniyama [26] and endomorphism algebra containing a CM subfield of $K$. The CM type of $A_{1, n+2}^{\prime}$ is given by

$$
\Phi_{0}=\Phi_{1, n+2}^{\prime}=\sum_{s \in(\mathbb{Z} / N \mathbb{Z})^{*}} r_{s}^{(0)} \sigma_{s}=\sum_{s \in T_{1, n+2}^{\prime}} \sigma_{s},
$$

where $T_{1, n+2}^{\prime}=T_{1-\mu_{1}, 1-\mu_{n+2}}$ and

$$
r_{s}^{(0)}=1-\left(\left\langle s\left(1-\mu_{1}\right)\right\rangle+\left\langle s\left(1-\mu_{n+2}\right)\right\rangle-\left\langle s\left(2-\mu_{1}-\mu_{n+2}\right)\right\rangle\right),
$$

with $r_{s}^{(0)}+r_{-s}^{(0)}=1$. The number $B\left(1-\mu_{1}, 1-\mu_{n+2}\right)$ is proportional over $\overline{\mathbb{Q}}$ to a non-zero period of $\omega_{1}(\mu ; \mathbf{0})$, which is a differential of the first kind, defined over $\overline{\mathbb{Q}}$, on $A_{1, n+2}^{\prime}$.

Similarly, we can construct the Prym variety $T_{N}=T_{N}(\mathbf{x})$ of $\mathcal{C}_{N}(\mathbf{x})$ for $\mathbf{x} \in \mathcal{Q}$. For proper divisors $f$ of $N$ there is a natural surjection from $\operatorname{Jac}\left(\mathcal{C}_{N}\right)$ to $\operatorname{Jac}\left(\mathcal{C}_{f}\right)$. Let $T_{N}=T_{N}(\mathbf{x})$ be the connected component of the origin in the intersection of all the kernels of these endomorphisms. The automorphism $\chi:(u, w) \mapsto\left(u, \zeta^{-1} w\right)$ of the affine model of $\mathcal{C}_{N}$ induces an action of $\zeta$ on $T_{N}$ which realizes the field $K$ in the endomorphism algebra $\operatorname{End}_{0}\left(T_{N}\right)=\operatorname{End}\left(T_{N}\right) \otimes \mathbb{Q}$ and an automorphism $\chi^{*}$ of the space of differentials of the first kind $H^{0}\left(T_{N}, \Omega\right)$ on $T_{N}$. For $s \in(\mathbb{Z} / N \mathbb{Z})^{*}$, let $V_{s}$ be the subspace of the elements of $H^{0}\left(T_{N}, \Omega\right)$ which are eigenspaces for the action of $K$ by $\chi^{*}$ with eigenvalue $\zeta^{s}$, that is, on which $K$ acts by $\sigma_{s}(K)$. An explicit basis for the subspaces $V_{s}, s \in(\mathbb{Z} / N \mathbb{Z})^{*}$ is given in [2]. The dimension of $V_{s}$ has been computed by several authors (see for example [4]) and equals,

$$
r_{s}^{(2)}=-1+\sum_{i=0}^{n+2}\left\langle s \mu_{i}\right\rangle .
$$

For all $s \in(\mathbb{Z} / N \mathbb{Z})^{*}$ we have $r_{s}^{(2)}+r_{-s}^{(2)}=(n+1)$. The space $H^{0}\left(T_{N}, \Omega\right)$ is the direct sum of the $V_{s}$; therefore the dimension of $T_{N}$ is $(n+1) \varphi(N) / 2$. We say that $T_{N}$ has (generalized) complex multiplication with (representation) type

$$
\Phi_{2}=\sum_{s \in(\mathbb{Z} / N \mathbb{Z})^{*}} r_{s}^{(2)} \sigma_{s} .
$$

The abelian variety $T_{N}$ is principally polarized.

Let $H_{\mu}$ be the subgroup of automorphisms $h \in(\mathbb{Z} / N \mathbb{Z})^{*}$ of $\Phi_{2}$ defined by the property

$$
h \Phi_{2}:=\sum_{s \in(\mathbb{Z} / N \mathbb{Z})^{*}} r_{h^{-1} s}^{(2)} \sigma_{s}=\Phi_{2} .
$$

The abelian variety $T_{N}$ is a power of an abelian variety $T_{N}^{\prime}$ whose endomorphism algebra contains the fixed field $K^{\prime}$ of $H_{\mu}$ in $K$ and has associated 
type

$$
\Phi_{2}^{\prime}=\sum_{s \in(\mathbb{Z} / N \mathbb{Z})^{*} / H_{\mu}} r_{s}^{(2)} \sigma_{s}
$$

where the sum is over a set of coset representatives of $H_{\mu}$ in $(\mathbb{Z} / N \mathbb{Z})^{*}$. The field $K^{\prime}$ is a CM field, except in the case when for all $s \in(\mathbb{Z} / N \mathbb{Z})^{*}$ we have $r_{s}^{(2)}=r_{-s}^{(2)}$. In that case $K^{\prime}$ is totally real. As $r_{1}^{(2)}=1$ and $r_{s}^{(2)}+r_{-s}^{(2)}=n+1$, this can only happen when $n=1$. This case is covered by [6], [8], [29], so we exclude it from now on.

Let $M$ be a set of representatives in $(\mathbb{Z} / N \mathbb{Z})^{*} /\{ \pm 1\}$ of those $s$ with $r_{s}^{(2)} r_{-s}^{(2)} \neq 0$. Let $m$ be the cardinality of $M$ and denote its elements by $r_{s_{j}}^{(2)}, j=1, \ldots, m$. For $s \in(\mathbb{Z} / N \mathbb{Z})^{*}$ and $\mathbf{x} \in \mathcal{Q}$, we let

$$
\omega^{(s)}=\omega^{(s)}(\mu ; \mathbf{x})=u^{-\left\langle s \mu_{0}\right\rangle}(u-1)^{-\left\langle s \mu_{1}\right\rangle} \prod_{i=2}^{n+1}\left(u-x_{i}\right)^{-\left\langle s \mu_{i}\right\rangle} d u .
$$

When $r_{s_{j}}^{(2)}=1$, the differential form $\omega^{\left(s_{j}\right)}$ is of the first kind and generates $V_{s_{j}}$. We can suppose that $s_{1}=1$, and therefore that $\omega^{\left(s_{1}\right)}=\omega_{1}=d u / w$. For $j=1, \ldots, m$, let $\left\langle s_{j} \mu\right\rangle$ be the rational ball $(n+3)$-tuple given by $\left\{\left\langle s_{j} \mu_{i}\right\rangle\right\}_{i=0}^{n+2}$. The Prym variety of $\mathcal{C}_{N}\left(\left\langle s_{j} \mu\right\rangle ; \mathbf{x}\right)$ is also $T_{N}(\mathbf{x})$. When $n=1$, since $r_{s}^{(2)}+r_{-s}^{(2)}=2$, the number $r_{s}^{(2)} r_{-s}^{(2)}$ equals 1 if it is non-vanishing. When $n=2$, since $r_{s}^{(2)}+r_{-s}^{(2)}=3$, the number $r_{s}^{(2)} r_{-s}^{(2)}$ equals 2 if it is non-vanishing. For $n \geq 3$, more cases are possible.

\section{Transcendence arguments}

We continue with the notations and assumptions of $\S 3$. For $\mathbf{x} \in \mathcal{Q} \cap \overline{\mathbb{Q}}^{n}$, the integral $\int_{1}^{\infty} \omega_{1}(\mu ; \mathbf{x})$ is either zero or proportional over $\overline{\mathbb{Q}}$ to a non-zero period of the first kind on the curve $\mathcal{C}_{N}$. By a corollary of the analytic subgroup theorem ([32], Theorem 5), non-zero periods of the first kind on curves defined over $\overline{\mathbb{Q}}$ are neither proportional over $\overline{\mathbb{Q}}$ to non-zero periods of the second kind nor to $\pi$. Therefore by (3.1) and the discussion of $\S 3$, for $\mu_{1}+\mu_{n+2} \leq 1$ the exceptional set $\mathcal{E}_{F}$ is empty.

We assume from now on that $\mu_{1}+\mu_{n+2}>1$. For two abelian varieties $A$ and $B$, we write $A \widehat{=} B$ when $A$ and $B$ are isogenous. We have the following corollary of the analytic subgroup theorem (see related arguments in [7], [8], [10], [29], for the cases $n=1,2$ and [11], [12] for the case $n \geq 3$.)

Proposition 4.1. If $\mathbf{x} \in \mathcal{E}_{F}$, then $T_{N}(\mathbf{x}) \hat{=} A_{1, n+2}^{\prime} \times D$, for an abelian variety $D$ with endomorphism algebra containing $K$ and of generalized $C M$ type $\Phi_{1}$ determined by the relation $\Phi_{2}=\Phi_{0}+\Phi_{1}$.

Proof: By [32], Theorem 5 (see also [23], Prop. 1, p6 and [22], Appendix), we deduce from (3.1) that if $\mathbf{x} \in \mathcal{E}_{F}$, then $T_{N}(\mathbf{x})$ and $A_{1, n+2}^{\prime}$ share, up to isogeny, a simple factor $B$. By the arguments of [3], §1, Exemple 3, the abelian variety $A_{1, n+2}^{\prime} \widehat{=} B^{s}$ is the smallest power of $B$ whose endomorphism 
algebra contains $K$. Moreover, we have $T_{N}(\mathbf{x}) \hat{=} B^{s t} \times C$ for some integer $t \geq 1$ and some abelian variety $C$ whose endomorphism algebra contains $K$ and which may be trivial. Therefore $T_{N}(\mathbf{x}) \widehat{=} A_{1, n+2}^{\prime} \times D$ for $D \widehat{=} B^{s(t-1)} \times C$. The generalized CM type $\Phi_{1}$ of $D$ must then be given by $\Phi_{1}=\Phi_{2}-\Phi_{0}$, as required.

Proposition 4.2. Suppose that the monodromy group $\Delta_{\mu}$ of $F=F_{\mu}(\mathbf{x})$ is an arithmetic lattice in $\mathrm{PU}(1, n)$. If $\mathbf{x} \in \mathcal{Q} \cap \overline{\mathbb{Q}}^{n}$ is not a zero of $F$, then $\mathbf{x} \in \mathcal{E}_{F}$ if an only if $T_{N}(\mathbf{x}) \hat{=} A_{1, n+2}^{\prime} \times A^{n}$, for an abelian variety $A$ of $C M$ type with endomorphism algebra containing $K$.

Proof: Suppose first that $\mathbf{x} \in \mathcal{E}_{F}$. As $r_{1}^{(2)}=r_{1}^{(0)}=1$ and $r_{s}^{(2)} r_{-s}^{(2)}=0$ for $s \in(\mathbb{Z} / N \mathbb{Z})^{*}, s \neq 1$, the type $\Phi_{1}$ is $n$ copies of a CM type $\Phi$. Therefore, we have $T_{N}(\mathbf{x}) \widehat{=} A_{1, n+2}^{\prime} \times A^{n}$ where $A$ is an abelian variety of CM type (see [25]). In particular $T_{N}(\mathbf{x})$ has $\mathrm{CM}$ (and not just generalized CM).

Conversely, suppose that $T_{N}(\mathbf{x}) \widehat{=} A_{1, n+2}^{\prime} \times A^{n}$, for an abelian variety $A$ of CM type. As $r_{1}^{(2)}=r_{1}^{(0)}=1$, the eigendifferentials $\omega_{1}(\mu ; \mathbf{x})$ and $\omega_{1}(\mu ; \mathbf{0})$ generate, on $T_{N}(\mathbf{x})$ and $A_{1, n+2}^{\prime}$ respectively, the 1-dimensional eigenspaces for the action of $K$ on the differentials of the first kind via the identity Galois embedding (see $\S 3$ ). Furthermore by [23], Proposition 5 , as $T_{N}(\mathbf{x})$ is of CM type, the non-zero periods of $\omega_{1}(\mu ; \mathbf{x})$ are proportional to each other over $\overline{\mathbb{Q}}$. Similarly, the non-zero periods of $\omega_{1}(\mu ; \mathbf{0})$ are proportional to each other over $\overline{\mathbb{Q}}$. In each case, the 1 -dimensional $\overline{\mathbb{Q}}$-vector space generated by these periods is an invariant of isogeny. Therefore (3.1) holds and so $\mathbf{x} \in \mathcal{E}_{F}$.

A converse of Proposition 4.1 also holds when $\Delta_{\mu}$ is not an arithmetic lattice, but we do not need it for the results of this paper.

We can assume in Proposition 4.1 that $T_{N}, D$, and $A_{1, n+2}^{\prime}$ are principally polarized and, as complex tori, have lattices isomorphic to the $\mathbb{Z}$-modules $M_{2}=\mathbb{Z}[\zeta]^{(n+1)}, M_{1}=\mathbb{Z}[\zeta]^{n}$ and $M_{0}=\mathbb{Z}[\zeta]$, respectively. For $i=0,1,2$, the data $\left(K, \Phi_{i}, M_{i}\right)$ determine a Shimura variety $S_{i}$. We can also assume that $\Phi_{2}$ is a generalized CM type with trivial automorphism group $H_{\mu}$ (see $\S 3)$, as we can always replace $\left(K, \Phi_{2}, M_{2}\right)$ by $\left(K^{\prime}, \Phi_{2}^{\prime}, M_{2}\right)$ in what follows. Writing

$$
\Phi_{i}=\sum_{s \in(\mathbb{Z} / N \mathbb{Z})^{*}} r_{s}^{(i)} \sigma_{s}, \quad i=0,1,2
$$

we have

$$
\operatorname{dim}\left(S_{i}\right)=\sum_{s \in(\mathbb{Z} / N \mathbb{Z})^{*} /\{ \pm 1\}} r_{s}^{(i)} r_{-s}^{(i)}
$$


The Shimura variety $S_{i}$ is the quotient of a complex symmetric domain $\mathcal{H}\left(\Phi_{i}\right)$ by an arithmetic (or modular) group $\Gamma_{i}$. We have,

$$
\mathcal{H}\left(\Phi_{i}\right)=\prod_{s \in(\mathbb{Z} / N \mathbb{Z})^{*} /\{ \pm 1\}} \mathcal{H}_{r_{s}^{(i)}, r_{-s}^{(i)}}, \quad i=0,1,2,
$$

where $\mathcal{H}_{u, v}$ is a point if $u v=0$ and, otherwise,

$$
\mathcal{H}_{u, v}=\left\{z \in M_{u, v}(\mathbb{C}): 1-z^{t} \bar{z} \quad \text { positive and hermitian }\right\} .
$$

When $s=1$ we have $r_{1}^{(2)}=1, r_{-1}^{(2)}=n$ and $\mathcal{H}_{1, n}$ is the complex $n$-ball $\mathbb{B}_{n}$. For details, see [25].

To every $\mathbf{x} \in \mathcal{Q}$, we can associate the point (or module) $P_{\mathbf{x}}$ in $S_{2}(\mathbb{C}$ ) which represents the isomorphism class of $T_{N}(\mathbf{x})$. Let $Z$ be the Zariski closure of $\left\{P_{\mathbf{x}}: \mathbf{x} \in \mathcal{Q}\right\}$; it is an irreducible closed subvariety of $S_{2}$ with dimension $\operatorname{dim}(Z)=n$.

By choosing as projective coordinates the $(n+1)$ elements of a suitable basis of solutions of the Appell-Lauricella system of partial differential equations $\mathcal{H}_{\mu}$, we can define a multi-valued developing map from $\mathcal{Q}$ to $\mathbb{P}_{n}(\mathbb{C})$ with image in $\mathcal{H}_{1, n}=\mathbb{B}_{n}$. For a choice of branch, the developing map sends $\mathbf{x} \in \mathcal{Q}$ to a point $z_{1} \in \mathbb{B}_{n}$. This point is the projection onto the first factor $\mathcal{H}_{1, n}$ of an element $z \in \mathcal{H}\left(\Phi_{2}\right)$ whose $\Gamma_{2}$-orbit corresponds to $P_{\mathbf{x}}$.

\section{Proof of Theorem 2.3}

Points in the same Hecke orbit on a Shimura variety correspond to abelian varieties in the same isogeny class. Therefore Propositions 4.1 and 4.2 are statements relating the exceptional set of $F$ to subsets of Hecke orbits of special subvarieties, which are special points in the zero dimensional case.

The first statement of the theorem was dealt with in $\S 4$. We assume therefore that $\mu_{1}+\mu_{n+2}>1$.

For the second statement of the theorem observe that, if $\Delta_{\mu}$ is arithmetic, then either $m=1$ or the restriction of scalars embedding of $\Delta_{\mu}$ in $\mathrm{PU}(1, n)^{m}$ is the identity map on $\Delta_{\mu}$ in each factor of $\mathrm{PU}(1, n)^{m}$. In this case, as explained in $\S 3$, we can factor out the CM-type by its automorphism group to reduce to the case $m=1$. We can therefore assume, and will assume, that $m=1$ in the arithmetic case. In the arithmetic case the variety $Z$ is a Shimura (hence special) variety. As Hecke orbits of special points are Zariski-dense in special varieties, the results of Proposition 4.2 imply that the set $\mathcal{E}_{F}$ is Zariski-dense in $Z$ and therefore in $\mathcal{Q}$.

It remains to prove the last statement of the theorem, namely the application of Conjecture 2.2 to $S=S_{2}$ and its subvariety $Z$ defined in $\S 4$. We assume from now on that $m>1$. The first step is to show that we can assume $S_{2}$ to be the special closure $Z$. The proper special subvarieties of $S_{2}$ are moduli spaces for abelian varieties whose endomorphism algebras strictly contain $K$. It is therefore enough to prove that for some $\mathbf{x} \in \mathcal{Q}$ the endomorphism algebra of $T_{N}(\mathbf{x})$ is exactly $K$. This is closely related to the result of [25], Theorem 5 for the Type IV case. Indeed, as we excluded in $\S 3$ 
the case $r_{s}^{(2)}=r_{-s}^{(2)}=1$, for all $s \in(\mathbb{Z} / N \mathbb{Z})^{*}$, and as we have assumed that the automorphism group of $\Phi_{2}$ is simple, it follows by the arguments of [25], $\S 4.6$ that we need only exhibit an element $\mathbf{x} \in \mathcal{Q}$ such that the center of the endomorphism algebra of $T_{N}(\mathbf{x})$ equals $K$. In the proof of Proposition 16 of that same reference, equation (49) gives conditions on the elements of $\mathcal{H}\left(\Phi_{2}\right)$ for their orbits under $\Gamma_{2}$ to correspond to abelian varieties whose endomorphism algebra has center strictly containing $K$. Whenever $r_{s}^{(2)} r_{-s}^{(2)} \neq 0$, $s \in(\mathbb{Z} / N \mathbb{Z})^{*}$, these conditions define an analytic subset $\mathcal{D}_{s}$ of $\mathcal{H}_{r_{s}^{(2)}, r_{-s}^{(2)}}$ of positive codimension. The image of $\mathcal{Q}$ in $\mathcal{H}_{1, n}=\mathbb{B}_{n}$ under a branch of the developing map (see the end of $\S 4$ ) has complex dimension $n$, and so intersects $\mathcal{D}_{1}$ in a set of positive codimension. Therefore, there is an $\mathbf{x} \in \mathcal{Q}$ whose image under the developing map is outside $\mathcal{D}_{1}$. The endomorphism algebra of $T_{N}(\mathbf{x})$ must then have center exactly $K$, as required. It follows that there is no proper special subvariety of $S_{2}$ containing $Z$, and so $S_{2}$ is the special closure of $Z$. It should also be possible to prove this by showing directly that, under the assumptions of this paragraph, the group $\Gamma_{2}$ is the smallest arithmetic group containing the monodromy group $\Delta_{\mu}$ of $F_{\mu}(\mathbf{x})$.

We can view the special variety $S_{1}$ as a special subvariety of $S_{2}$ by associating to the module of $D \in S_{1}$ the module of $A_{1, n+2}^{\prime} \times D$ in $S_{2}$. Recall from $\S 3$ that $m$ is the number of $s \in(\mathbb{Z} / N \mathbb{Z})^{*}$ with $r_{s}^{(2)} r_{-s}^{(2)} \neq 0$, and that we denoted them by $s_{j}, j=1, \ldots, m$. As $\Phi_{1}=\Phi_{2}-\Phi_{0}$ we have $r_{1}^{(1)}=0$ and therefore,

$$
\operatorname{dim}\left(S_{1}\right)=\sum_{j=2}^{m} r_{s_{j}}^{(1)} r_{-s_{j}}^{(1)},
$$

where $r_{ \pm s_{j}}^{(1)}=r_{ \pm s_{j}}^{(2)}-1$ if $r_{ \pm s_{j}}^{(0)}=1$. Clearly, from (5.1), as $m>1$,

$$
\operatorname{dim}\left(S_{1}\right)<\operatorname{dim}\left(S_{2}\right)-r_{1}^{(2)} r_{-1}^{(2)}=\operatorname{dim}\left(S_{2}\right)-n=\operatorname{dim}\left(S_{Z}\right)-\operatorname{dim}(Z) .
$$

Therefore, by Proposition 4.1 and Conjecture 2.2, the last statement of Theorem 2.3 follows.

By way of example, let us see how (5.1), (5.2) look for the case $n=2$, $m>1$. As $r_{s}^{(2)} r_{-s}^{(2)}=0$ or 2 for all $s \in(\mathbb{Z} / N \mathbb{Z})^{*}$, we have $\operatorname{dim}\left(S_{2}\right)=2 m$. On the other hand, we have

$$
\Phi_{1}=\sum_{s \in(\mathbb{Z} / N \mathbb{Z})^{*}} r_{s}^{(1)} \sigma_{s}
$$

where $r_{s}^{(1)}+r_{-s}^{(1)}=2$. Since $r_{1}^{(1)}=0$, and $r_{s}^{(1)} r_{-s}^{(1)}=0$ whenever $r_{s}^{(2)} r_{-s}^{(2)}=0$, we have $\operatorname{dim}\left(S_{1}\right)=m^{\prime} \leq(m-1)<2(m-1)=\operatorname{dim}\left(S_{2}\right)-2$.

\section{Proof of Theorem 2.4 and Desrousseaux's conditions}

We continue with the notation of $\S \S 3,4$, and 5. Suppose now that $n=2$ and that the monodromy group $\Delta_{\mu}$ is a lattice in $\mathrm{PU}(1,2)$. Assume that 
$\mu$ is a rational ball 5-tuple with $\mu_{1}+\mu_{4}>1$. By [7], Lemme 1 and 2, p.676, with $\mu_{0}, \mu_{2}$ replaced by $\mu_{1}, \mu_{4}$, we have $\left\langle s \mu_{1}\right\rangle+\left\langle s \mu_{4}\right\rangle>1$ for all $s \in(\mathbb{Z} / N \mathbb{Z})^{*}$ with $r_{s}^{(2)}=1$. Therefore for all $s \in(\mathbb{Z} / N \mathbb{Z})^{*}$ with $r_{s}^{(2)}=1$, we have $r_{s}^{(0)}=1$ and therefore

$$
r_{s}^{(1)}=0, \quad r_{-s}^{(1)}=2,
$$

since $\Phi_{1}=\Phi_{2}-\Phi_{0}$. Therefore $\operatorname{dim}\left(S_{1}\right)=0$ and the abelian variety $D$ of Proposition 4.1 must always be isogenous to the square $A^{2}$ of an abelian variety $A$ of CM type. (Compare with the arguments of Théorème 2 of [7].) The points of $\mathcal{E}_{F}$ are then special points in a single Hecke orbit. Hence the André-Oort conjecture can be used in place of Conjecture 2.2.

This means that, in this case, the extra conditions in the definition of the exceptional set appearing in Théorème of [10] are unnecessary. However, when the monodromy group is not a lattice, they may indeed be necessary, as the examples in $\S 7$ show. Indeed, these extra conditions force the abelian variety $D$ to be isogenous to an abelian variety of CM type.

\section{Some EXAMPles IN THE CASE $n=2$}

Example 1: Consider the rational ball 5 -tuple

$$
\mu=\left(\frac{3}{12}, \frac{7}{12}, \frac{3}{12}, \frac{5}{12}, \frac{6}{12}\right) .
$$

The corresponding monodromy group $\Delta_{\mu}$ is a non-arithmetic lattice in $\mathrm{PU}(1,2)$ and is number 18 in the list in [9]. In this case, $N=12$ and $r_{1}^{(2)}=1, r_{-1}^{(2)}=2, r_{5}^{(2)}=1, r_{-5}^{(2)}=2$. We have $\mu_{1}+\mu_{4}=13 / 12>1$ and

$$
\langle 5 \mu\rangle=\left(\frac{3}{12}, \frac{11}{12}, \frac{3}{12}, \frac{1}{12}, \frac{6}{12}\right),
$$

so that $\left\langle 5 \mu_{1}\right\rangle+\left\langle 5 \mu_{4}\right\rangle=11 / 12+6 / 12=17 / 12>1$. Therefore,

$$
\Phi_{2}=\sigma_{1}+2 \sigma_{-1}+\sigma_{5}+2 \sigma_{-5}, \quad \Phi_{1}=2 \sigma_{-1}+2 \sigma_{-5}, \quad \Phi_{0}=\sigma_{1}+\sigma_{5} .
$$

Example 2: Consider the rational ball 5 -tuple,

$$
\mu=\left(\frac{29}{105}, \frac{101}{105}, \frac{1}{105}, \frac{41}{105}, \frac{38}{105}\right) .
$$

By [15], Lemma 5, the corresponding monodromy group $\Delta_{\mu}$ is not a lattice in PU $(1,2)$, since $N=105>84$. We have $\mu_{1}+\mu_{4}=139 / 105>1$. On the other hand, for $s=52 \in(\mathbb{Z} / N \mathbb{Z})^{*}$, we have

$$
\langle 52 \mu\rangle=\left(\frac{38}{105}, \frac{17}{105}, \frac{52}{105}, \frac{17}{105}, \frac{86}{105}\right) .
$$

Therefore $\left\langle 52 \mu_{1}\right\rangle+\left\langle 52 \mu_{4}\right\rangle=103 / 105<1$ and so $r_{52}^{(0)}=0$. It follows that $r_{52}^{(1)}=r_{52}^{(2)}-r_{52}^{(0)}=1, r_{-52}^{(1)}=1$ and so $S_{1}$ has positive dimension by (4.2). 


\section{REFERENCES}

[1] Appell, P., Kampé de Fériet, M.J.: Fonctions hypergéométriques et hypersphériques. Polynômes d'Hermite, Gauthier-Villars 1926.

[2] Archinard, N.: Hypergeometric abelian varieties. Canad. J. Math. 55 (5) (2003), 897932.

[3] Bertrand, D.: Endomorphismes de groupes algébriques; applications arithmétiques. In: Approximations diophantiennes et nombres transcendants, Prog. Math. 31, pp. 1-45 1983.

[4] Chevalley, Cl., Weil, A.: Uber das Verhalten der Integrale 1. Gattung bei Automorphismen des Funktionkörpers. Abh. Hamburger Math. Sem. 10 (1934), 358-361.

[5] Cohen, P.B.: Humbert surfaces and transcendence properties of automorphic functions. Rocky Mountain J. Math., 26 (3) (1996), 987-1001.

[6] Cohen, P.B., Wolfart, J: Modular embeddings for some non-arithmetic Fuchsian groups. Acta Arithmetica, LVI (1990), 93-110.

[7] Cohen, P.B., Wolfart, J.: Fonctions hypergéométriques en plusieurs variables et espaces des modules de variétés abéliennes. Ann. scient. Éc. Norm. Sup., 4e série, t.26, (1993), 665-690.

[8] Cohen, P.B., Wüstholz, G: Application of the André-Oort conjecture to some questions in transcendence. In: Panorama of Number Theory, A view from Baker's garden (ed. by G. Wüstholz), pp. 89-106. Cambridge U. Press, Cambridge 2002.

[9] Deligne, P., Mostow, G.D.: Monodromy of hypergeometric functions and nonlattice integral monodromy. Publ. I.H.E.S. 63 (1986), 5-89.

[10] Desrousseaux, P-A.: Valeurs exceptionnelles de fonctions hypergéométriques d'Appell. Ramanujan J. 8 (3) (2004), 331-355.

[11] Desrousseaux, P-A: Fonctions hypergéométriques de Lauricella, périodes de variétés abéliennes et transcendance. Comptes rendus mathém. de l'Acad. des Sciences, La Soc. royale de Canada 26 (4) (2004), 110-117.

[12] Desrousseaux, P-A: Exceptional sets of Lauricella hypergeometric functions. Journal of Algebra, Number Theory and Applications, Vol. 5 (3) (2005), 429-467.

[13] Desrousseaux, P-A., Tretkoff, M.D., Tretkoff, P., Transcendence of values at algebraic points for certain higher order hypergeometric functions, IMRN/15278 (61) 2005, $3835-3854$

[14] Edixhoven, S., Yafaev, A: Subvarieties of Shimura varieties. Annals of Math. 157 (2003), 621-645.

[15] Felikson, A.A.: On Thurston signatures. (Russian) Uspekhi Mat. Nauk. 52 (4) (1997), 217-218; translation in Russian Math. Surveys 52 (4) (1997), 826-827.

[16] Koblitz, N., Rohrlich, D.: Simple factors in the Jacobian of a Fermat curve, Can. J. Math. 30 (1978), 1183-1205.

[17] Mostow, G.D.: Generalized Picard lattices arising from half-integral conditions, Publ. de l'I.H.E.S. 63 (1986), 91-106.

[18] Noot, R.: Correspondances de Hecke, action de Galois et la Conjecture d'André-Oort [d'après Edixhoven et Yafaev]. Séminaire Bourbaki, 57ème année, 2004/5, Exp. No. 942 (novembre 2004).

[19] Pink, R.: A Combination of the Conjectures by Mordell-Lang and André-Oort. In: Geometric Methods in Algebra and Number Theory (Bogomolov, F., Tschinkel, Y., Eds.) Progress in Math. 235, Basel: Birkhäuser (2005), 251-282.

[20] Pink, R.: A Common Generalization of the Conjectures of André-Oort. ManinMumford, and Mordell-Lang, preprint, April 17, 2005.

[21] Sauter, J.K.: Isomorphisms among monodromy groups and applications to lattices in PU $(1,2)$. Pacific J. Math. 146 (1990), 331-384. 
[22] Shiga, H., Tsutsui, T., Wolfart, J.: Triangle Fuchsian differential equations with apparent singularities (with an appendix by P. B. Cohen). Osaka J. Math. 41 (2004), 625-658.

[23] Shiga, H., Wolfart, J: Criteria for complex multiplication and transcendence properties of automorphic functions. J. Reine Angew. Math. 463 (1995), 1-25.

[24] Shiga, H., Wolfart, J.: Algebraic values of Schwarz triangle functions. Technical Reports of Math. Sciences Chiba Univ. 20 (5) 2004.

[25] Shimura, G: On analytic families of polarized abelian varieties and automorphic functions. Annals of Math. 78 (1963), 149-192.

[26] Shimura, G., Taniyama, Y: Complex multiplication of abelian varieties and its applications to number theory. Publ. Math. Soc. Japan 6, 1961.

[27] Takeuchi, K: Arithmetic triangle groups. J. Math. Soc. Japan 29 (1977), 91-106.

[28] Yoshida, M: Fuchsian differential equations. Aspects of math., Vieweg, 1987.

[29] Wolfart, J: Werte hypergeometrische Funktionen. Invent. Math. 92 (1988), 187-216.

[30] Wolfart, J., Wüstholz, G: Der Überlagerungsradius gewisser algeraische Kurven und die Werte der Betafunktionen an rationalen Stellen. Math. Ann. 273 (1985), 1-15.

[31] Wüstholz, G.: Zum Periodenproblem. Invent. Math. 78 (1984), 381-391.

[32] Wüstholz, G.: Algebraic groups, Hodge theory and transcendence. In: Proc. of the Intern. Congress of Math. 1, pp 476-483, Berkeley, California, USA, 1986.

[33] Wüstholz, G: Algebraische Punkte auf analytischen Untergruppen algebraischer Gruppen, Annals of Math. 129 (1989), 501-517.

Current address: Department of Mathematics, Texas A\&M University, TAMU 3368, TX 77843-3368, USA, and UMR 8524 au CNRS, Université des Sciences et Technologies de Lille, Villeneuve d'Ascq, 59655, FRANCE.

All correspondence to go to: Paula Tretkoff, Department of Mathematics, Texas A\&M University, TAMU 3368, TX 77843-3368, USA, ptretkoff@math.tamu.edu E-mail address: Pierre-Antoine.Desrousseaux@math.univ-lille1.fr

E-mail address: tretkoff@math.tamu.edu

E-mail address: ptretkoff@math.tamu.edu 